\section{ANTIBIOTIC DISCOVERY}

\section{Macrolides en masse Nature 533, 338-345 (2016)}

Macrolide antibiotics exhibit potent activity against human pathogens, but generating synthetic and engineered biosynthetic routes to new members of this class has proven difficult. All of the macrolide antibiotics currently approved for use in humans have been manufactured through semisynthesis from erythromycin, a strategy that is restricted by the limited functional groups on the molecule that can be easily modified and by the challenge of selectively modifying them. Seiple et al. report a new synthetic approach to macrolide production involving a highly convergent assembly process from simple building blocks, which allows the diversification of macrolide structures through incorporation of a wide variety of functional groups. Using this system, the authors prepared over 300 macrolide compounds from the azaketolide and ketolide subclasses, including the approved drug telithromycin and the clinical candidate solithromycin. When the compound library was tested against a panel of bacterial strains, the majority exhibited antibiotic activity, and two compounds in particular were found to be more potent than any other macrolide currently in clinical use for the treatment of antibiotic-resistant pathogens. This synthetic approach provides a potentially important new strategy for the development of new antibiotics needed to combat the advance of antibiotic resistance and a tool to optimize their properties for use in humans.
RNA STRUCTURE

\section{PARISian arches \\ Cell 165, 1267-1279 (2016)}

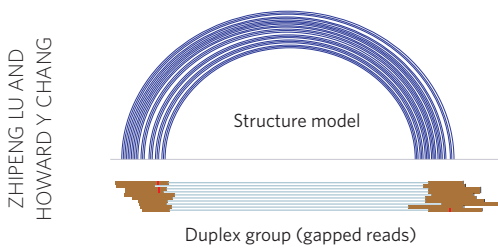

Base-paired duplexes are the fundamental unit of RNA structure and RNA-RNA interactions. Chemical probing and informatics tools that define paired and unpaired regions have enabled RNA structural analyses within cells, but these approaches generally uncover only shortrange interactions and simple RNA motifs. Lu et al. now report PARIS (psoralen analysis of RNA interactions and structures) as a method for mapping RNA duplexes on a transcriptome-wide scale. The approach relies on UV-mediated psoralen crosslinking of duplex regions in cells followed by analysis of the isolated crosslinked fragments by reverse transcription and next-generation DNA sequencing (NGS). 'Duplex groups' are then assigned by informatics analysis to infer candidate intramolecular stem-loop structures or in trans RNA-RNA interactions within cells. PARIS applied to several human and mouse cell lines revealed that duplex formation between regions separated by hundreds or thousands of nucleotides is widespread in the transcriptome. Further, the crossspecies comparisons and multiple NGS reads per locus identified more complex
$\mathrm{Ca}_{\mathrm{v}} 1.3$ channels are voltage-gated channels that allow the passage of $\mathrm{Ca}^{2+}$ ions into cells, including hippocampal neurons, and recent studies have raised the possibility that $\mathrm{Ca}^{2+}$ influx is facilitated by interactions between neighboring $\mathrm{Ca}_{\mathrm{v}}$ channels. From monitoring $\mathrm{Ca}^{2+}$-dependent facilitation (CDF), a regulatory mechanism induced by $\mathrm{Ca}_{v} 1.3$ channel opening, in a human cell line, Moreno et al. found that whereas the short $\mathrm{Ca}_{v} 1.3$ channel splice variant, $\mathrm{Ca}_{v} 1.3_{S}$, was subject to $\mathrm{CDF}$, the long form, $\mathrm{Ca}_{v} 1.3_{L}$, was not. By TIRF microscopy, $\mathrm{Ca}_{\mathrm{v}} 1.3_{\mathrm{s}}$-expressing cells showed greater numbers of multiquantal $\mathrm{Ca}^{2+}$-influx events than $\mathrm{Ca}_{\mathrm{v}} 1 . \mathrm{B}_{\mathrm{L}}$-expressing cells did, suggesting a cooperative gating mechanism whereby multiple $\mathrm{Ca}_{\mathrm{v}} 1.3_{\mathrm{s}}$ channels are active simultaneously. Super-resolution microscopy followed by step-photobleaching and optogenetic experiments revealed that $\mathrm{Ca}_{\mathrm{v}} 1.3_{\mathrm{S}}$ clusters in hippocampal neurons, with a mean of eight channels per cluster, and the channels physically interact within the clusters. $\mathrm{Ca}^{2+}$ binding to calmodulin ( $\mathrm{CaM}$ ) was required for the channel coupling, which increased the firing rate in hippocampal neurons. These results point to a mechanism where $\mathrm{Ca}_{\mathrm{v}} 1.3_{\mathrm{s}}$ undergoes cooperative gating through functional coupling supported by $\mathrm{CaM}$ association with the channels, ultimately leading to regulation of neuronal excitability through amplification of $\mathrm{Ca}^{2+}$ signals. structures (such as pseudoknots) and abundant alternative RNA conformations between duplex groups. Application of PARIS with other probing methods yielded a detailed duplex map of Xist, providing structural evidence that A-repeat assembly in this lncRNA is essential for binding of SPEN, a factor involved in X-chromosome inactivation. PARIS adds to the RNA toolbox for mapping RNA structure within cells and exploring its functional roles.

METHANOGENESIS

\section{A radical approach \\ Science 352, 953-958 (2016)}

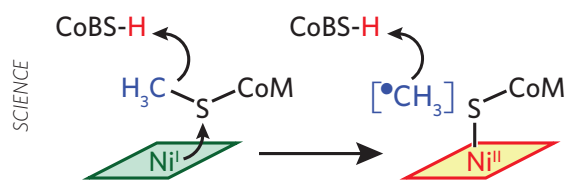

Over $90 \%$ of Earth's methane is produced by the enzyme methyl-coenzyme M (methylSCoM) reductase (MCR) in methanogenic archaea. Using a nickel cofactor in the active site, MCR converts methyl-SCoM and coenzyme $\mathrm{B}(\mathrm{CoBSH})$ to methane and the mixed disulfide CoBS-SCoM. Two mechanisms have been proposed for this reaction, distinguished by their expected first intermediates: an organometallic methyl-Ni(III) complex or a methyl radical. To slow the reaction, enabling accumulation and observation of the intermediate, Wongnate et al. used a CoBSH analog with a shorter side chain. Through a combination of stopped-flow studies, rapid chemical and freeze quenching, and spectroscopy, the authors characterized this key intermediate and the kinetics of the reaction. These experiments, supported by quantum chemical calculations and molecular dynamics simulations, led them to propose a reaction mechanism wherein the first step consists of $\mathrm{Ni}(\mathrm{I})$ attack on methyl-SCoM to generate a methyl radical and a Ni(II)-thiolate complex. The methyl radical then abstracts a hydrogen atom from $\mathrm{CoBSH}$, producing methane and the CoBS• radical, which combines with the $\mathrm{Ni}$ (II)bound thiolate. The greater understanding of the enzyme mechanism afforded by this study should benefit the development of technologies to generate and activate methane, which is an energy source as well as a greenhouse gas. 